\title{
Antioxidant Activities Correlation Analysis of Procyanidins from China Cultivars Litchi Pericarp
}

\author{
Weijing Zhou ${ }^{1,2}$, Xiaoming Jiang ${ }^{1, *}$, Ping He ${ }^{1}$, Zhida Sun ${ }^{2}$, Bijun Xie $^{2}$ \\ ${ }^{1}$ Wuhan Institute for Food and Cosmetic Control, Wuhan, PR China \\ ${ }^{2}$ Department of Food Chemistry and Molecular Biology, College of Food Science and Technology, Huazhong Agricultural University, Wuhan, \\ PR China
}

Email address:

elfinlife221@163.com (Xiaoming Jiang)

${ }^{*}$ Corresponding author

\section{To cite this article:}

Weijing Zhou, Xiaoming Jiang, Ping He, Zhida Sun, Bijun Xie. Antioxidant Activities Correlation Analysis of Procyanidins from China Cultivars Litchi Pericarp. Science Journal of Analytical Chemistry. Vol. 7, No. 1, 2019, pp. 27-31. doi: 10.11648/j.sjac.20190701.14

Received: February 13, 2019; Accepted: March 18, 2019; Published: April 3, 2019

\begin{abstract}
Ethanol extracts of litchi pericarp procyanidins (LPPC) were purified from 7 Chinese litchi cultivars. The procyanidin contents in LPPC were ranged from $659.7 \mathrm{mg}$ to $1163.2 \mathrm{mg}$ grape seed procyanidins/g equivalent, while the concentrations of (-)-epicatechin, procyanidin dimmer and trimer were further investigated as well. Antioxidation and free radical scavenging activities of LPPC from different sources were evaluated and compared in vitro. The results showed that LPPC for 'Huaizhi' owned the highest free radical scavenging activity on hydrogen peroxide $\left(\mathrm{H}_{2} \mathrm{O}_{2}\right)$, superoxide radical $\left(\mathrm{O}_{2}^{\circ-}\right)$ and ferric reducing; 'Feizixiao' exhibited the highest scavenging capacity on 2,2-diphenyl-1-picrylhydrazyl radical (DPPH·), hydroxyl radical ( $\mathrm{OH})$ and lipid peroxidation, however, 'Guiwei' possessed the highest value of antioxidant activity based on $\beta$-carotene bleaching and ferrous chelating assay. Furthermore, the compositions of LPPC suggested positive correlations with their antioxidant activities significantly.
\end{abstract}

Keywords: Litchi Cultivars, Pericarp, Procyanidins, Oligomer, Antioxidant Activity

\section{Introduction}

Litchi (Litchi chinensis Sonn.), which is a tropical fruit of the Sapindaceae family originating from South-east Asia, is a white aril surrounded by a bright red attractive pericarp. Since 2005, litchi cultivated area was more than $6 \times 10^{5}$ hectare, and its yield was more than $1.3 \times 10^{6}$ ton per year, of which Guangdong province denominated 'The Flora of Guangdong Litchi'[1]. They are Guiwei, Feizixiao, Jinfeng, Sanyuehong, Heiye Nuomici and Huaizhi, respectively. The names of litchi are based upon the shape of tortoiseshell-like cracking segments the pericarp, the shape of leaves, inflorescence and fruit, maturity time and the quality of fruit [2].

Litchi extracts have attracted attention in the field of nutrition and pharmaceutical value due to their various active compounds such as anthocyanins, flavanols, phenolic and polysaccharide in aril, flower, seed and pericarp. These compounds have exhibited a dose-dependent on free-radical-scavenging activity [3]. In our previous studies, the main oligomeric procyanidins (such as epicatechin- $(4 \beta \rightarrow 8,2 \beta \rightarrow \mathrm{O} \rightarrow 7)$-epicatechin,

epicatechin- $(4 \beta \rightarrow 8,2 \beta \rightarrow \mathrm{O} \rightarrow 7)$-epicatechin- $(4 \beta \rightarrow 8)$-epicatech in) from litchi pericarp were isolated and identified [4]. However, there are still limited publishing studies concerning a completed antioxidant properties of the procyanidins extracts from pericarp of different litchi cultivars in vitro. Therefore, the objective of present study was to analyze the procyanidin compositions of seven well-known litchi pericarp extracts by HPLC method, and then evaluate their antioxidant and free radicals scavenging activities. The relation of the antioxidant activities of LPPC and the procyanidins content in litchi pericarp extract were also discussed, which are essential for predicting their bioactivities and subsequent end-use in food.

\section{Material and Methods}

\subsection{Plant Materials}

Seven litchi (Litchi Chinensis Soon.) cultivars, at 
commercial maturation were obtained from Guangdong province in China. These litchi cultivars named 'Sanyuehong', 'Feizixiao', 'Guiwei', 'Nuomici', 'Heiye', 'Huaizhi' and 'Jinfeng' (Figure 1) were collected according to the representative and the fruit maturation period (from May to August). All cultivars litchi were freshly-pickinged fruits. The fruit arrived in the laboratory within $24 \mathrm{~h}$ of harvest. Fruits were peeled by handwork and their pericarps were stored at $-20^{\circ} \mathrm{C}$ for further use.

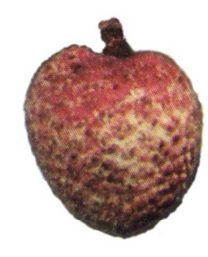

Sanyuehong
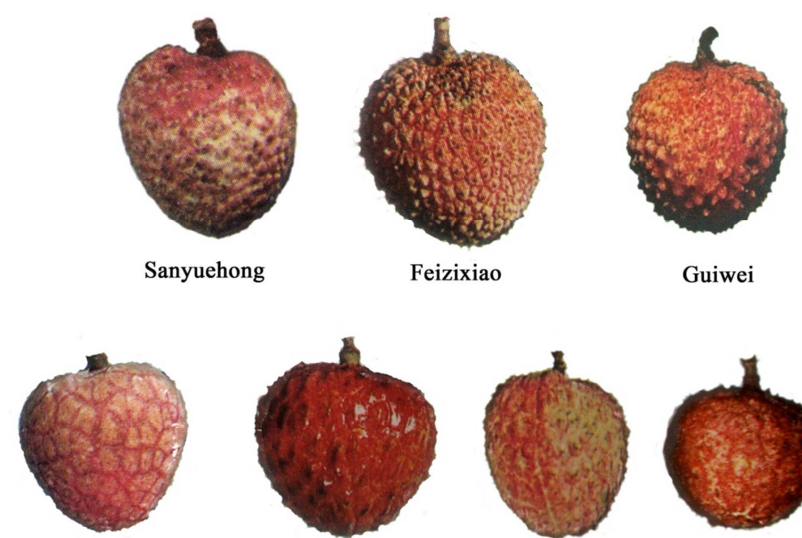

Heiye

$4 \mathrm{~cm}$

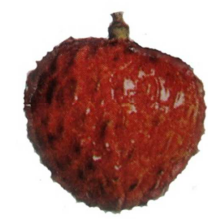

Nuomici

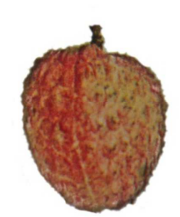

Jingfeng
Huaizhi

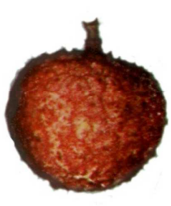

Figure 1. Samples of seven well known cultivars litchi.

\subsection{Chemicals and Reagents}

Chemicals and reagents were obtained from the following commercial sources: (-)-epicatechin, DPPH, $\beta$-carotene, luminal, deoxyribose and pyrogallol were purchased from Sigma Chemical Co. (St. Louis, MO, USA). The grape seed procyanidins standarad was obtained from Jianfeng Co., Ltd (Tianjin, PRC). AB-8 resin was produced by Nankai Hecheng Science \& technology Co., Ltd (Tianjin, PRC). A MDA (Malondialdehyde) kit was purchased from Nanjing Jiancheng Institute of Biology and Engineering (Nanjing, PRC). All other chemicals were of analytical grade.

\subsection{Extraction and Purification of Procyanidins}

Frozen litchi pericarp (50g) was extracted using 70\% ethanol $(750 \mathrm{~mL})$ in a water bath at $70{ }^{\circ} \mathrm{C}$ for $2 \mathrm{~h}$. The extract was filtered through a vacuum filtering system. The filtrates were concentrated under vacuum by a rotary evaporator at $50^{\circ} \mathrm{C}$. The concentrated sample $(250 \mathrm{~mL})$ was loaded onto an AB- 8 resin column $(30 \times 2.5 \mathrm{~cm}, \mathrm{ID} ; 25-40 \mu \mathrm{m}$ particle size $)$, and the fraction eluted by ethanol-water $(80: 20, \mathrm{v} / \mathrm{v})$, and was collected at a flow rate of $3 \mathrm{~mL} / \mathrm{min}$. The eluates were evaporated under vacuum and the residue was lyophilized to obtain LPPC.

\subsection{Determination of Procyanidins Content}

\subsubsection{Butanol-HCl Assay}

The method used to determine the contents of procyanidins were according to $\mathrm{Li}$ [5]. The grape seed procyanidins was used as standard to calculate the LPPC content by the equation

of $\mathrm{Y}=4.1268 \mathrm{X}+0.0161, \mathrm{R}^{2}=0.9995$ from the standard curve. The LPPC content was expressed as the grape seed procyanidins equivalents (GSPC) in $\mathrm{mg} / \mathrm{g}$ of fresh-frozen weight.

\subsubsection{Reversed-Phase High-Performance Liquid Chromatography (RP-HPLC) Analysis}

The analysis of procyanidins of litchi pericarp extract by RP-HPLC was performed on a Varian liquid chromatogram, and detection was carried out using a photodiode array detector. The chromatographic separation was carried out on a column VP-ODS column $(150 \mathrm{~mm} \times 4.6 \mathrm{~mm}$ ID, $5 \mu \mathrm{m}$ particle size, SHIMADZU, Japan). The column oven temperature was set at $20^{\circ} \mathrm{C}$ throughout the whole analytical procedure. The mobile phase was delivered at a flow rate of $1.0 \mathrm{~mL} / \mathrm{min}$ consisting of $0.4 \% \mathrm{v} / \mathrm{v}$ aqueous acetic acid (A) and acetonitrile (B). A $10 \mu \mathrm{l}$ sample solution was injected and the elution gradient program was as follows: $0-15 \mathrm{~min}, 5 \%-15 \% \mathrm{~B} ; 15-45$ min, $15 \%-50 \% \mathrm{~B}$. The column was then equilibrated with $5 \%$ $\mathrm{B}$ for $5 \mathrm{~min}$ before the next injection. The absorbance of the eluates was monitored at $280 \mathrm{~nm}$. Before being injected, the extracts were filtered through a $0.45 \mu \mathrm{m}$ millipore membrane.

The procyanidins trimer epicatechin- $(4 \beta \rightarrow 8,2 \beta \rightarrow \mathrm{O} \rightarrow 7)$ epicatechin- $(4 \beta \rightarrow 8)$ - epicatechin and A2 dimmer can be identified according to compare with the retention time of the standard afforded by our lab in RP-HPLC chromatographic peak from our laboratory authentic analytic results and publication paper $[4,6]$. It can evaluate the concentration of trimer and A2 dimmer according to compare the peak area percentage.

\subsubsection{Antioxidant Activity Determination}

Antioxidative properties of procyanidins contributed to their capacities on free radical scavenging, metal ion chelating and reducing, lipid peroxidation inhibition. The purpose of this experiment is to evaluate and compare the "in vitro" antioxidant activity of seven cultivars LPPC in different aspect according to use the following assays. DPPH free radical scavenging assay [4]. DPPH radical scavenging activity was assessed according to the method of Shimada. $\beta$-Carotene-linoleate bleaching assay. The scavenging ability of LPPC on hydroxide peroxide was measured by a chemiluminescence (CL) method in a luminol- $\mathrm{H}_{2} \mathrm{O}_{2}$ system by BPLC-4 Ultra-weak luminescence Analyzer [7]. Hydrogen peroxide scavenging assay. The scavenging ability of LPPC on hydroxide peroxide was measured by a chemiluminescence (CL) method in a luminol- $\mathrm{H}_{2} \mathrm{O}_{2}$ system by BPLC-4 Ultra-weak luminescence Analyzer [8]. Hydroxyl radical scavenging assay. The hydroxyl radical $(\mathrm{OH})$ scavenging ability was determined according to the method [9]. Superoxide anion radical scavenging assay. The scavenging activities of superoxide free radical were determined with some modifications [10]. Ferrous metal ion chelating assay. The ferrous-chelating ability of LPPC was determined according to the method described Suter [11]. Ferric ion reducing assay $\left(\mathrm{Fe}^{3+}\right.$ to $\left.\mathrm{Fe}^{2+}\right)$. The ferric ion reducing capacity was determined according to the method of Wang [12]. Inhibition of lipid peroxidation assay. The inhibition of lipid 
peroxidation of LPPC was evaluated using MDA kit [13]. Statistical analysis. Statistical analysis was done with SPSS 13.0 using a one-way analysis of variance.

\section{Results and Discussion}

\subsection{Isolation and Determination of LPPC}

The RP-HPLC analysis of LPPC showed 3 mainly compounds eluting between 15 and $40 \mathrm{~min}$ (Figure 2). Most of those compounds had a maximal absorbance wavelength at $280 \mathrm{~nm}$, corresponding to the characteristic absorbance spectrum of flavan-3-ols. Compound 1 was tentative identified as (-)-epicatechin by co-injection with standards, the compound 2 and 3 were identified as procyanidin trimer epicatechin- $(4 \beta \rightarrow 8, \quad 2 \beta \rightarrow O \rightarrow 7)$-epicatechin- $(4 \beta \rightarrow 8)$ epicatechin and procyanidin A2 dimmer respectively, according to the laboratory authentic analytic results and paper published [4]. The chromatograms of 7 LPPC were similar, in which (-)-epicatechin, A-type dimmer and trimer were dominant, but differed from peak intensity.

The contents of procyanidins from 7 LPPC was listed in Table 1. The higher contents were found in 'Huaizhi' 'Feizixiao' and 'Guiwei', and the lowest content was observed in 'Jinfeng'. Furthermore, (-)-epicatechin content in LPPC was examined. 'Guiwei' showed the highest value, followed by 'Huaizhi' and 'Feizixiao'. The (-)-epicatechin content detected in 'Jinfeng' was also the lowest. However, differences occurred in A-type dimmer content, 'Feizixiao' was $14.16 \%$, which was slightly higher than 'Huaizhi' and 'Guiwei', and the others were less than $10 \%$. We supposed that 'Huaizhi' and 'Feizixiao' LPPC might be of interest to be used as alternative cultivars for preparing antioxidants.

The LPPC content, yield, pericarp weight rate and harvest time as shown in Table 1. It was found that higher LPPC content also have the higher yield. LPPC content decreased with the harvest time delay in order except 'Huaizhi'. However, LPPC content has not showed a significant correlation with the color and the shape of litchi pericarp (Figure 1).

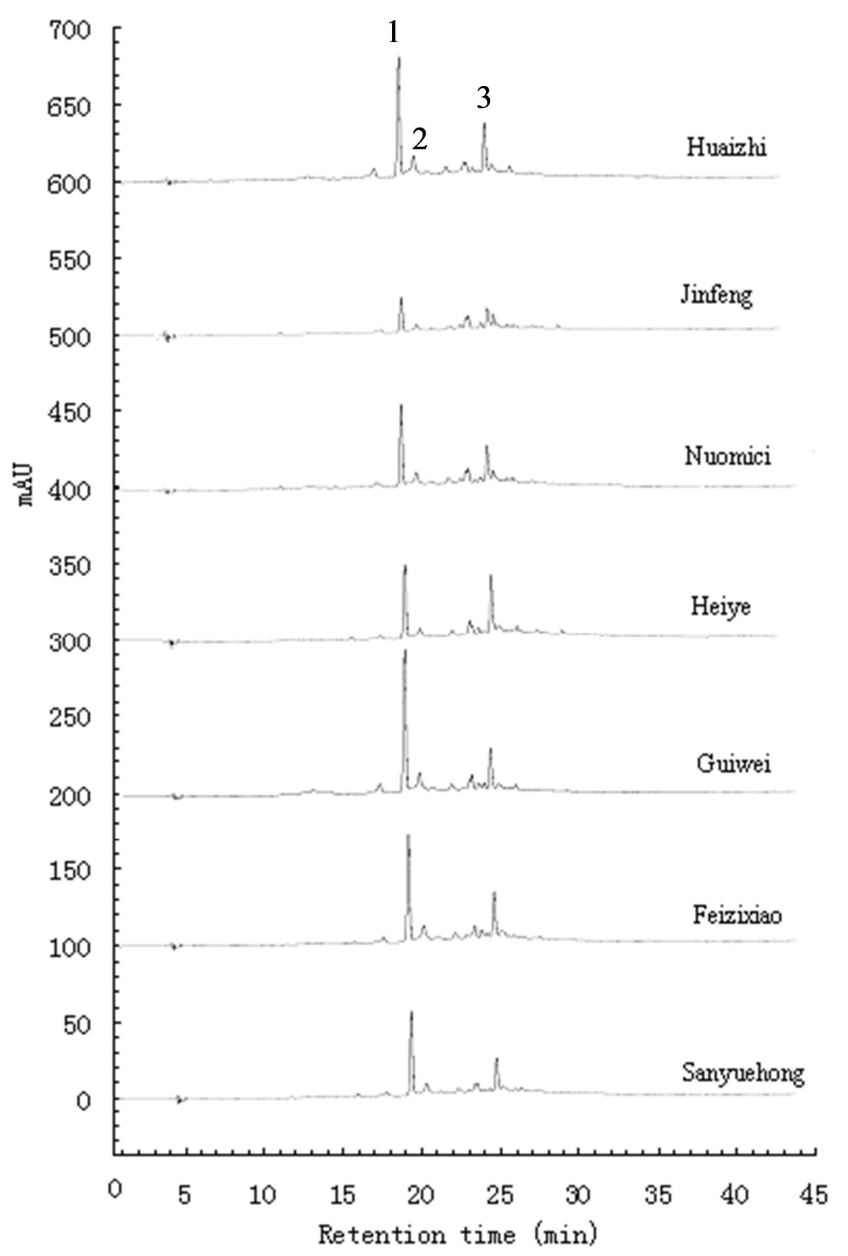

Figure 2. HPLC chromatographic profile of seven cultivars $L P P C$.

1: (-)- Epicatechin; 2: Trimeric procyanidins; 3: A-type dimmer. Solvent A $0.4 \%$ aq. acetic acid, solvent B acetonitrile. Linear gradients: $0-15 \mathrm{~min}$, $5-15 \% \mathrm{~B} ; 15-45 \mathrm{~min}, 15-50 \% \mathrm{~B}$. Flow rate was $1 \mathrm{~mL} / \mathrm{min}$, detection at 280 $\mathrm{nm}$, VP-ODS column $(5 \mu \mathrm{m})$.

Table 1. Nine litchi cultivars pericarps characteristics and procyanidins contents.

\begin{tabular}{|c|c|c|c|c|c|c|c|}
\hline $\begin{array}{l}\text { Cultivar } \\
\text { name }\end{array}$ & Maturity time & $\begin{array}{l}\text { Pericarp weight } \\
\text { rate (\% of fresh } \\
\text { fruit) }\end{array}$ & $\begin{array}{l}\text { Yield (\% of fresh } \\
\text { pericarp) }\end{array}$ & $\begin{array}{l}\text { Procyanidins } \\
\text { Contents (mg } \\
\text { GSPC/ g extracts) }^{\text {a }}\end{array}$ & (-)-Epicatechin ${ }^{\mathrm{b}}(\%)$ & $\begin{array}{l}\text { A-type } \\
\text { dimmer }^{\mathrm{b}}(\%)\end{array}$ & $\begin{array}{l}\text { Trimeric } \\
\text { procyanidins }^{\mathrm{b}} \\
(\%) \\
\end{array}$ \\
\hline Sanyuehong & Middle May & $20.94 \pm 2.9$ & $1.02 \pm 1.7$ & $895.7 \pm 9.9$ & $30.34 \pm 0.93$ & $13.23 \pm 0.23$ & $9.25 \pm 0.07$ \\
\hline Feizixiao & Early June & $20.25 \pm 5.1$ & $1.30 \pm 1.2$ & $1076.1 \pm 14.8$ & $29.01 \pm 0.46$ & $14.16 \pm 0.14$ & $10.58 \pm 0.16$ \\
\hline Guiwei & Middle June & $17.31 \pm 17$ & $1.40 \pm 1.7$ & $1075.2 \pm 10.5$ & $35.97 \pm 0.73$ & $5.25 \pm 0.12$ & $10.01 \pm 0.06$ \\
\hline Heiye & Late June & $14.91 \pm 2.3$ & $0.95 \pm 1.2$ & $827.5 \pm 15.6$ & $16.28 \pm 0.59$ & $6.08 \pm 0.06$ & $5.91 \pm 0.08$ \\
\hline Nuomici & early July & $17.67 \pm 5.9$ & $0.80 \pm 1.0$ & $697.9 \pm 16.3$ & $16.34 \pm 0.64$ & $3.89 \pm 0.14$ & $6.27 \pm 0.12$ \\
\hline Jinfeng & early July & $16.17 \pm 1.6$ & $0.63 \pm 1.6$ & $659.7 \pm 23.4$ & $11.39 \pm 0.91$ & $2.81 \pm 0.36$ & $6.03 \pm 0.23$ \\
\hline Huaizhi & Middle July & $17.01 \pm 2.9$ & $1.51 \pm 2.0$ & $1163.2 \pm 141$ & $31.21 \pm 0.48$ & $13.96 \pm 0.08$ & $13.24 \pm 0.18$ \\
\hline
\end{tabular}

a Procyanidins contents expressed as mg of grape seed procyanidins equivalents/g litchi pericarp extracts.

b (-)-Epicatechin, Trimeric procyanidins and A-type dimmer contents expressed as area percent of epicatechin peak in HPLC chromatogram.

\subsection{Comparative Antioxidant Activity of LPPC}

Antioxidation and free radical scavenging activities of LPPC from different sources were evaluated and compared in these tests. Figure 3 showed the free radical scavenging activites of 7 cultivars LPPC at various concentrations. and
Table 2 showed the effective half inhibition concentration $\left(\mathrm{IC}_{50}\right)$ of LPPC and ascorbic acid on DPPH radicals. The proocyanidins significantly inhibited the activity of free radicals in a dose-dependent manner. The results showed that LPPC for 'Huaizhi' owned the highest free radical scavenging activity on hydrogen peroxide $\left(\mathrm{H}_{2} \mathrm{O}_{2}\right)$, superoxide radical 
$\left(\mathrm{O}_{2}^{\bullet-}\right)$ and ferric reducing; 'Feizixiao' exhibited the highest scavenging capacity on 2,2-diphenyl-1-picrylhydrazyl radical $(\mathrm{DPPH} \cdot)$, hydroxyl radical $(\mathrm{OH})$ and lipid peroxidation,
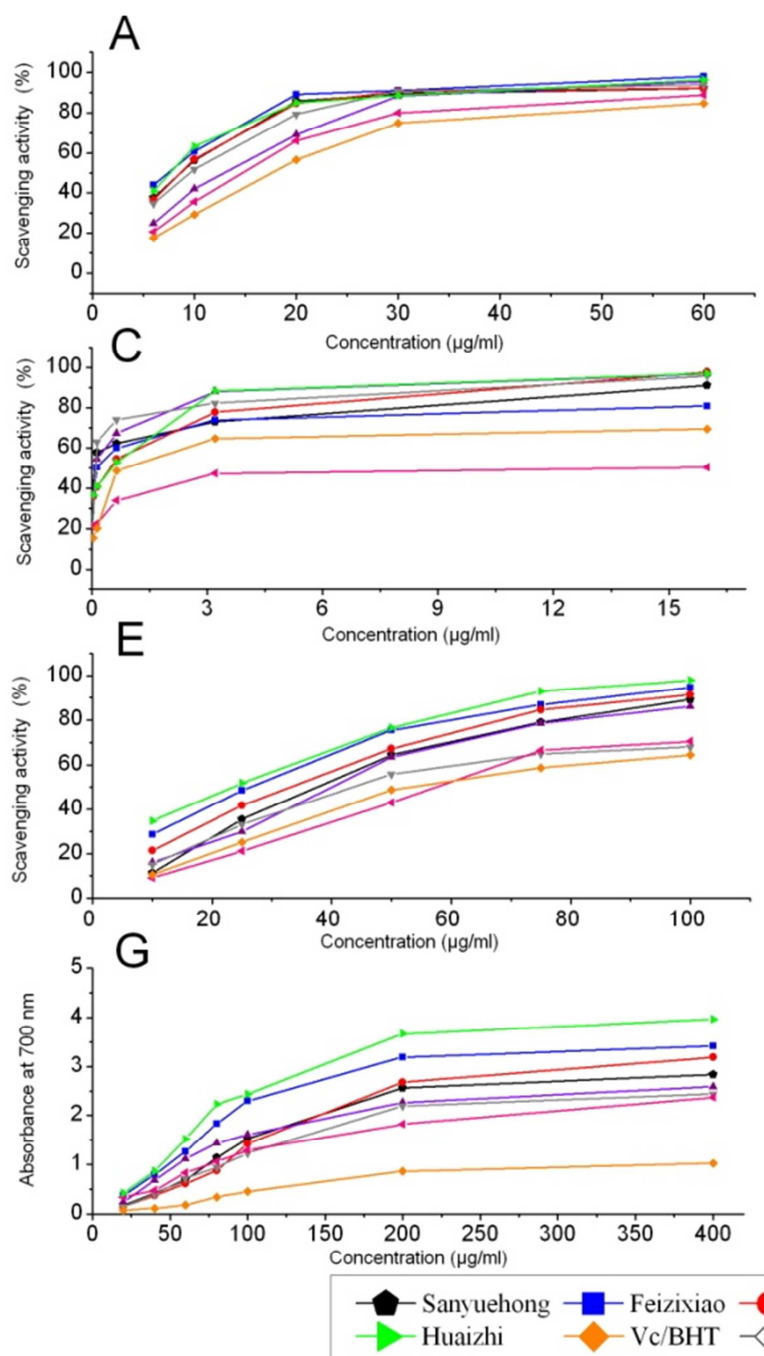

however, 'Guiwei' possessed the highest value of antioxidant activity based on $\beta$-carotene bleaching and ferrous chelating assay.
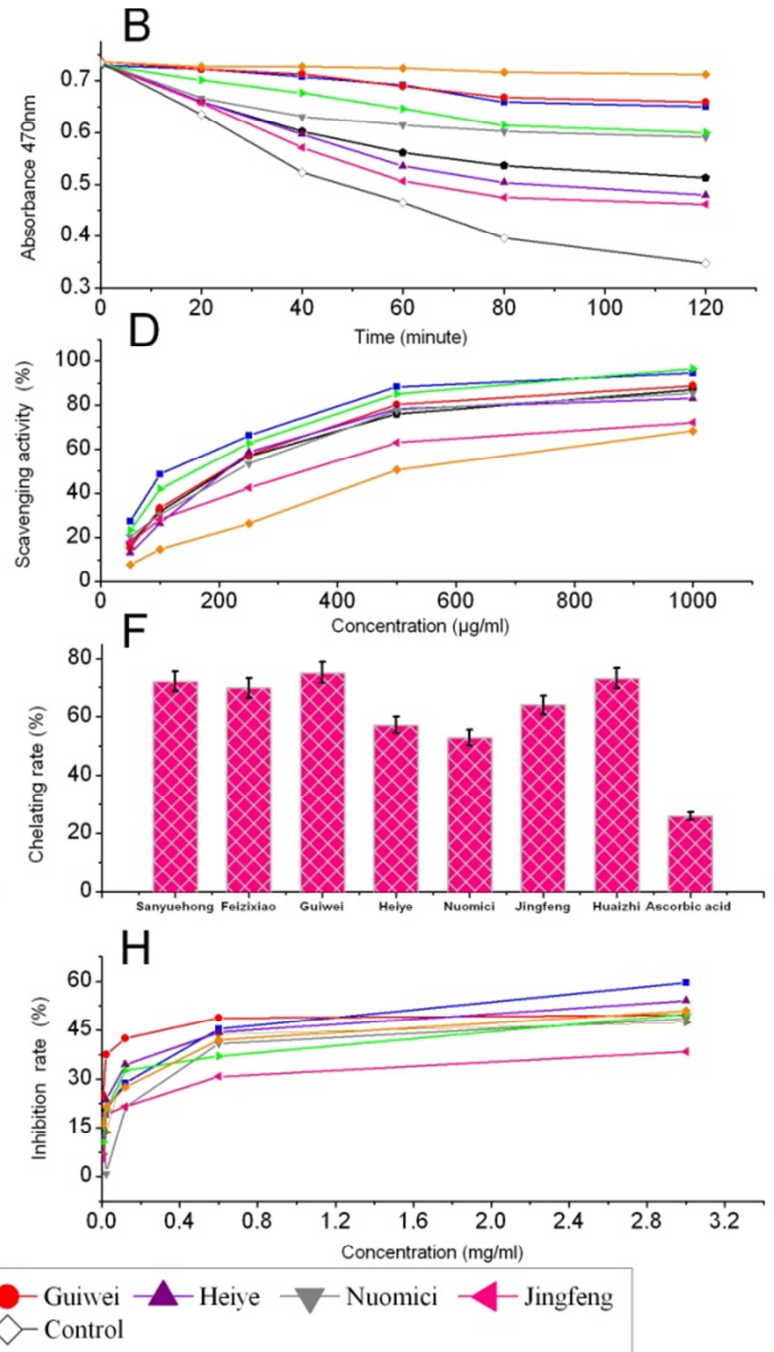

Figure 3. Antioxidant activity of seven cultivars LPPC at different system.

A: DPPH radical scavenging activity. B: Quenching singlet oxygen ability. C: Decomposition hydrogen peroxide ability. D: Scavenging hydroxyl radical ability. E: Scavenging superoxide anion radical ability. F: Chelating metal ion ability. G: Reducing metal ion ability (evaluate by absorbance at $\mathrm{A}_{700}$ ). $\mathrm{H}$ : Inhibition of lipid peroxidation ability.

Table 2. Antioxidant activity of seven cultivars LPPC and standard antioxidants ascorbic acid and BHT in different aspect.

\begin{tabular}{|c|c|c|c|c|c|c|c|c|}
\hline \multirow[b]{2}{*}{ Varieties } & \multicolumn{5}{|c|}{ Scavenging free radicals } & \multirow{2}{*}{$\begin{array}{l}\begin{array}{l}\text { Chelating } \\
\text { ferric ion }\end{array} \\
\text { (mg EDTA/g } \\
\text { sample) }\end{array}$} & \multirow{2}{*}{$\begin{array}{l}\text { Reducing } \\
\text { ferric ion }\end{array}$} & \multirow{2}{*}{$\begin{array}{l}\begin{array}{l}\text { Inhibit lipid } \\
\text { peroxidant }\end{array} \\
\text { MDA content (nmol } \\
\text { /mgprot }\end{array}$} \\
\hline & $\begin{array}{l}\text { DPPH } \\
\text { IC }_{50}(\mu \mathrm{g} / \mathrm{mL})\end{array}$ & $\begin{array}{l}{ }^{1} \mathrm{O}_{2} \\
\text { ACC }\end{array}$ & $\begin{array}{l}\mathrm{H}_{2} \mathrm{O}_{2} \\
\mathrm{IC}_{50}(\mu \mathrm{g} / \mathrm{mL})\end{array}$ & $\begin{array}{l}\mathrm{OH} \cdot \\
\mathrm{IC}_{50}(\mu \mathrm{g} / \mathrm{mL})\end{array}$ & $\begin{array}{l}\mathrm{O}_{2}^{--} \\
\mathrm{IC}_{50}(\mu \mathrm{g} / \mathrm{mL})\end{array}$ & & & \\
\hline Sanyuehong & $9.02 \pm 0.93$ & $426.36 \pm 17.14$ & $0.227 \pm 0.013$ & $260.76 \pm 6.13$ & $42.15 \pm 1.56$ & $17.12 \pm 1.04$ & $2.83 \pm 0.15$ & $2.33 \pm 0.15$ \\
\hline Feizixiao & $7.32 \pm 1.12$ & $782.94 \pm 16.25$ & $0.165 \pm 0.006$ & $162.69 \pm 15.71$ & $28.93 \pm 0.95$ & $15.30 \pm 0.61$ & $3.42 \pm 0.12$ & $1.78 \pm 0.08$ \\
\hline Guiwei & $9.18 \pm 0.69$ & $806.20 \pm 12.69$ & $0.191 \pm 0.004$ & $249.98 \pm 8.54$ & $36.11 \pm 2.04$ & $21.28 \pm 0.97$ & $3.18 \pm 0.16$ & $1.99 \pm 0.07$ \\
\hline Heiye & $10.27 \pm 3.18$ & $338.50 \pm 25.27$ & $0.283 \pm 0.002$ & $265.81 \pm 11.26$ & $44.78 \pm 3.20$ & $13.55 \pm 1.02$ & $2.58 \pm 0.04$ & $1.83 \pm 0.11$ \\
\hline Nuomici & $14.15 \pm 1.58$ & $630.49 \pm 23.69$ & $0.325 \pm 0.013$ & $296.24 \pm 12.54$ & $49.95 \pm 0.63$ & $13.37 \pm 0.92$ & $2.44 \pm 0.08$ & $2.36 \pm 0.10$ \\
\hline Jinfeng & $14.85 \pm 1.83$ & $294.57 \pm 21.56$ & $0.364 \pm 0.005$ & $350.67 \pm 13.96$ & $56.01 \pm 1.19$ & $14.01 \pm 1.56$ & $2.37 \pm 0.05$ & $2.41 \pm 0.09$ \\
\hline Huaizhi & $7.58 \pm 0.94$ & $651.16 \pm 23.84$ & $0.148 \pm 0.009$ & $199.51 \pm 9.25$ & $23.03 \pm 1.75$ & $18.52 \pm 1.30$ & $3.96 \pm 0.10$ & $2.34 \pm 0.03$ \\
\hline $\begin{array}{l}\text { Positive } \\
\text { control }\end{array}$ & $18.75 \pm 2.27^{\mathrm{b}}$ & $952.88 \pm 18.96^{\mathrm{c}}$ & $0.692 \pm 0.013^{\mathrm{b}}$ & $408.34 \pm 9.01^{\mathrm{b}}$ & $58.91 \pm 0.73^{\mathrm{b}}$ & $13.01 \pm 0.69^{\mathrm{b}}$ & $1.03 \pm 0.09^{\mathrm{b}}$ & $2.46 \pm 0.17^{\mathrm{c}}$ \\
\hline
\end{tabular}

${ }^{a}$ Values are the means of there replicates \pm SD.

${ }^{\mathrm{b}}$ Using ascorbic acid as the positive control.

${ }^{\mathrm{c}}$ Using BHT as the positive control. 


\subsection{Correlation Analysis}

In these studies, the Pearson correlation coefficient for relationship between the content of procyanidins or oligomeric procyanidins and antioxidant activities from different litchi cultivars named 'Sanyuehong', 'Feizixiao', 'Guiwei', 'Nuomici', 'Heiye', 'Huaizhi' and 'Jinfeng' are shown in Table 3. The excellent correlations were found between procyanidins content and $\mathrm{IC}_{50}$ on scavenging $\mathrm{DPPH}$, $\mathrm{H}_{2} \mathrm{O}_{2}, \mathrm{OH}, \mathrm{O}_{2}^{\circ-}$ or $\mathrm{A}_{700}$ of reducing ferric ion $\left(\mathrm{r}^{2}=-0.925\right.$, $-0.984,-0.879,-0.970$ and 0.952 , respectively), so was the trimer $\left(r^{2}=-0.820,-0.929,-0.790,-0.935\right.$ and 0.973 , respectively). At the same time, good correlations were found for A-type dimmer content and $\mathrm{IC}_{50}$ on scavenging $\mathrm{DPPH}$,
$\mathrm{H}_{2} \mathrm{O}_{2}, \mathrm{OH}, \mathrm{O}_{2}^{\bullet-}$ or $\mathrm{A}_{700}$ of reducing ferric ion $\left(\mathrm{r}^{2}=-0.846\right.$, $-0.807,-0.826,0.796$ and 0.756 , respectively). However, a correlation appeared between the (-)-epicatechin content and the scavenging $\mathrm{H}_{2} \mathrm{O}_{2}$ ability and chelating ferric ion rate with excellent correlation coefficient $\left(\mathrm{r}^{2}=-0.919,0.886\right), \mathrm{IC}_{50}$ on scavenging $\mathrm{DPPH} \cdot$ and $\mathrm{A}_{700}$ of reducing ferric ion with negative correlation coefficient $\left(\mathrm{r}^{2}=-0.840,-0.819\right)$. But there was no correlation between either the LPPC content or the ${ }^{1} \mathrm{O}_{2}$ scavenging data and lipid peroxidation activities as for the interaction with biomembrane. Overall, the contributions to antioxidant activities were mainly attributed to procyanidins and its oligomeric procyanidins contents.

Table 3. Correlations coefficients of the procyanidins compounds to antioxidant activities in different system.

\begin{tabular}{|c|c|c|c|c|c|c|c|c|}
\hline & $\begin{array}{l}\text { DPPH } \\
\text { (Scavenging } \\
\text { rate) }\end{array}$ & $\begin{array}{l}{ }^{1} \mathrm{O}_{2} \\
\text { (Scavenging } \\
\text { rate) }\end{array}$ & $\begin{array}{l}\mathrm{H}_{2} \mathrm{O}_{2} \\
\text { (Scavenging } \\
\text { rate) }\end{array}$ & $\begin{array}{l}\mathrm{OH} \cdot \\
\text { (Scavenging } \\
\text { rate) }\end{array}$ & $\begin{array}{l}\mathrm{O}_{2}^{\bullet-} \\
\text { (Scavenging } \\
\text { rate) }\end{array}$ & $\begin{array}{l}\text { Rate } \\
\text { (Chelating } \\
\text { ferric ion) }\end{array}$ & $\begin{array}{l}\text { A }_{700} \\
\text { (Reducing } \\
\text { ferric ion) }\end{array}$ & $\begin{array}{l}\text { MDA content } \\
\text { (lipid } \\
\text { proxidantion) }\end{array}$ \\
\hline procyanidins & $-0.925^{* *}$ & 0.698 & $-0.984^{* *}$ & $-0.879^{* *}$ & $-0.970^{* *}$ & 0.751 & $0.952^{* *}$ & 0.384 \\
\hline epicatechin & $-0.840^{*}$ & 0.706 & $-0.919^{* *}$ & -0.724 & $-0.819^{*}$ & $0.886^{* *}$ & $0.801 *$ & 0.102 \\
\hline A-type dimmer & $-0.846^{*}$ & 0.323 & $-0.807^{*}$ & $-0.826^{*}$ & $0.796^{*}$ & 0.303 & $0.756^{*}$ & -0.164 \\
\hline trimmer & $-0.820^{* *}$ & 0.639 & $-0.929^{* *}$ & $-0.790^{*}$ & $-0.935^{* *}$ & 0.727 & $0.973^{* *}$ & -0.056 \\
\hline
\end{tabular}

** Correlations significant at a 0.01 level (1-tailed).

* Correlations significant at a 0.05 level (1-tailed)

\section{Conclusions}

The present study strongly suggested that litchi is a new plant source of A-type procyanidins and natural antioxidant, and highlighted the antioxidation dose-effect relationship effect of the litchi pericarp, which have been regarded as waste in the past. More specifically, procyanidin oligomers in litchi pericarp were recorded from 7 Chinese litchi cultivars, 'Huaizhi' and 'Feizixiao' LPPC, compared to other well known cultivars, showed the highest level of antioxidant and free radical scavenging activities based on different methods in vitro. The results also showed that the antioxidant activities partially depend on the procyanidins composition and cultivars, therein 'Huaizhi', 'Feizixiao' were the optimal cultivars for preparing nature antioxidant.

\section{References}

[1] L. Yuan, J. Gan, and Z. Bi. (2007). The advancement of research on litchi and longan germplasm resources in China. Scientia Horticulturae, 114, 143-150.

[2] L. Shuyi., J. Xiao, L. Chen, and C. Hu, (2012). Identification of A-series oligomeric procyanidins from pericarp of Litchi chinensis by FT-ICR-MS and LC-MS. Food Chemistry, 135, 31-38.

[3] X. Duan, Y. Jiang, and G. Xin (2007). Antioxidant properties of anthocyanins extracted from litchi fruit pericarp tissues in relation to their role in the pericarp browning. Food Chemistry, $101,1365-1371$.

[4] L. Liang, X. Bijun, and C. Shaoqian. (2007). A-type procyanidins from litchi chinensis pericarp with antioxidant activity. Food Chemistry, 5, 1446-1451.
[5] L. Shuyi, L. Chen, and T. Yang (2013). Increasing antioxidant activity of procyanidin extracts from the pericarp of litchi chinensis processing waste by two probiotic bacteria bioconversions. Journal of Agricultural and Food Chemistry, 61, 2506- 2512.

[6] L. Shuyi., Y. Liu, and G. Liu. (2017). Effect of the A-type linkage on the pharmacokinetics and intestinal metabolism of litchi pericarp oligomeric procyanidins. Journal of Agricultural and Food Chemistry, 65, 1893-1899.

[7] E. Conde, C. Cara, and A. Moure (2009). Antioxidant activity of the phenolic compounds released by hydrothermal treatments of olive tree pruning. Food Chemisry, 114, 806-812.

[8] G. Shanshan, D. Qianchun, and J. Xiao (2007). Evaluation of antioxidant activity and preventing DNA damage effect of pomegranate extracts by chemiluminescence method. Journal of Agricultural and Food Chemistry, 55, 3134-3140.

[9] Q. Wu, S. Li and J. Xiao (2017). Analysis of distribution and pharmacokinetics of litchi pericarp procyanidins in rat plasma and organs by using liquid chromatography-tandem mass spectrometry. European Food Research and Technology, 243, $167-176$.

[10] A. Rechner, C. Kroner (2005). Anthocyanins and colonic metabolites of dietary polyphenols inhibit platelet function. Thrombosis Research, 116, 327-334.

[11] M. Suter, Richter C. (2010). Anti and pro-oxidative properties of PADMA Tibetan herbal formulation. Redox Report, 5, 17-22.

[12] L. Wang, J. Yen, and H. Ling (2013). Antioxidant effect of methanol extracts from lotus plumule and blossom. Journal of Food and Drug Analysis, 11, 60-66.

[13] J. Li, and Jiang Y. M. (2007). Litchi flavonoids: isolation, identification and biological activity. Molecules, 12, 745-758. 